Wilfrid Laurier University

Scholars Commons @ Laurier

8-2009

\title{
Core-Offset Small-Core-Diameter Dispersion Compensation Fiber Interferometer and its Applications in Fiber Sensors
}

\author{
Bo Dong \\ Wilfrid Laurier University \\ Da-Peng Zhou \\ University of Waterloo \\ Wing-Ki Liu \\ University of Waterloo \\ John W.Y. Lit \\ Wilfrid Laurier University, jlit@wlu.ca
}

Follow this and additional works at: https://scholars.wlu.ca/phys_faculty

\section{Recommended Citation}

Dong, Bo; Zhou, Da-Peng; Liu, Wing-Ki; and Lit, John W.Y., "Core-Offset Small-Core-Diameter Dispersion Compensation Fiber Interferometer and its Applications in Fiber Sensors" (2009). Physics and Computer Science Faculty Publications. 10.

https://scholars.wlu.ca/phys_faculty/10

This Article is brought to you for free and open access by the Physics and Computer Science at Scholars Commons @ Laurier. It has been accepted for inclusion in Physics and Computer Science Faculty Publications by an authorized administrator of Scholars Commons @ Laurier. For more information, please contact scholarscommons@wlu.ca. 


\title{
Core-offset small-core-diameter dispersion compensation fiber interferometer and its applications in fiber sensors
}

\author{
Bo Dong, ${ }^{1, *}$ Li Wei, ${ }^{1,2}$ Da-Peng Zhou, ${ }^{2}$ Wing-Ki Liu, ${ }^{2}$ and John W. Y. Lit ${ }^{1}$ \\ 'Department of Physics and Computer Science, Wilfrid Laurier University, Waterloo, Ontario, N2L 3C5, Canada \\ ${ }^{2}$ Department of Physics and Astronomy, Guelph-Waterloo Physics Institute, University of Waterloo, \\ Waterloo, Ontario N2L 3G1, Canada \\ *Corresponding author: dbo1978@163.com
}

Received 24 February 2009; revised 9 July 2009; accepted 27 July 2009;

posted 28 July 2009 (Doc. ID 107941); published 4 August 2009

\begin{abstract}
We propose a core-offset small core diameter dispersion compensation fiber (DCF) interferometer and investigate its applications in fiber sensors. If the transverse force is applied to a short section of the DCF, there is almost no crosstalk on the transmission spectrum between the extinction ratio variation induced by the transverse force and the wavelength shift caused by the longitudinal strain or ambient temperature, which can be applied to measure both transverse and longitudinal strain, or both transverse strain and temperature, simultaneously. The proposed sensors have the advantages of low cost, simple and compact structure, and good reproducibility. (C) 2009 Optical Society of America

OCIS codes: $\quad 060.2310,060.2370$.
\end{abstract}

\section{Introduction}

Multimode fiber interferometers (MMIs) based on intermodal interference have attracted much attention for their advantages of low cost and simple structure. Several MMI sensors [1-5], focusing on one-directional (longitudinal [1- $\overline{4}]$ or transverse [5]) strain sensing, have been reported. However, it is highly desirable to measure more than onedirectional strain for modern structural applications. Several multidirectional strain sensors $[6,7]$ have been reported, such as using a planar fiber optic sensor array [6], or forming a single mode fiber (SMF) Mach-Zehnder interferometer (MZI) [7] where three sensing arms are needed. However, each of them has to use more than two sensing heads and has a very complex structure, which increases the cost of the system and limits the real world application. Moreover, it is also highly desirable to realize multiparameter measurement in today's industry, but most of

0003-6935/09/234577-05\$15.00/0

(C) 2009 Optical Society of America the reported MMI sensors [1-3,5] are focused on oneparameter measurement. Although simultaneous measurement of strain and temperature with an MMI [4] has been achieved, the MMI has to be connected a fiber Bragg grating, which leads to a complex structure and high cost. So a simple and compact sensor with only one sensing head to realize multidirectional strain measurement and multiparameter measurement is highly attractive.

In this paper, we propose a core-offset smallcore-diameter dispersion compensation fiber (DCF) interferometer and investigate its applications in fiber sensors. We found that this type of interferometer can be effectively used as a simple and compact sensor with only one sensing head to realize simultaneous measurement of transverse and longitudinal strain or simultaneous measurement of transverse strain and temperature. The DCF in the interferometer was originally designed for dispersion compensation applications, with a core/cladding diameter of $1.9 \mu \mathrm{m} / 115.7 \mu \mathrm{m}$, a large dispersion parameter of $-270 \mathrm{ps} / \mathrm{nm} / \mathrm{km}$ at $1550 \mathrm{~nm}$, and a cutoff wavelength of $1663 \mathrm{~nm}$. It is claimed as a multimode 
fiber because the fact of the cutoff wavelength at $1663 \mathrm{~nm}$ makes it naturally a multimode fiber at wavelengths shorter than $1663 \mathrm{~nm}$. By core offsetting a splice between the DCF and a SMF, an interferometer with a high extinction ratio can be obtained. This extinction ratio will change with the variations of the transverse force applied to a short section of the DCF, while the interference phase will remain almost constant. Moreover, it is almost independent of the longitudinal strain variation applied to the whole DCF or temperature variations, which can be applied to measure both transverse and longitudinal strain, or both transverse strain and temperature, simultaneously. The sensors have the advantages of low cost, simple and compact structure, and good reproducibility.

\section{Principle}

A. Structure and Characteristics of the Interferometer

Figure 1 shows the experimental setup. A C-band unpolarized broadband source (BBS) was used as the light source. The transmission spectrum of the interferometer is measured by an optical spectrum analyzer (OSA). The interferometer is formed by about a $22.5 \mathrm{~cm}$ DCF sandwiched between two SMFs (SMF28 type), which serves as a Mach-Zehdner interferometer based on intermodal interference. The extinction ratio for the DCF interferometer without core offset is generally small. In order to obtain a high extinction ratio, we slightly offset a splice between the DCF and the SMF. The extinction ratio of typical interference spectrum shown in Fig. 1 is more than $9 \mathrm{~dB}$. It can be seen that the interference is mainly produced by two dominant interference modes, which indicates that other existing modes are carrying less power compared to the two interference modes.

In order to study the interference modes' type, several core-offset DCF interferometers were fabricated with the cladding layer of the DCF being stripped. After the bare DCFs were immersed in the indexmatch liquid, the transmission spectra remain unchanged, which confirmed that the interference modes are core modes. The extinction ratio of the interferometer can be approximately expressed as

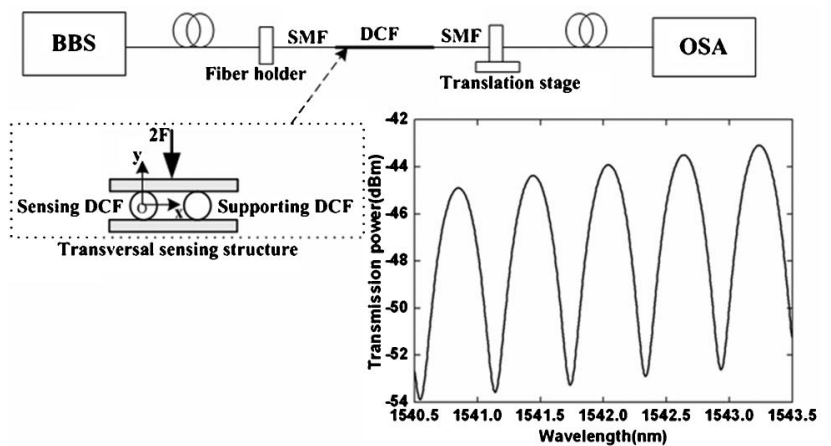

Fig. 1. Experimental setup and transmission spectrum of the interferometer (the inset shows the transverse sensing structure). extinction ratio $=10 \lg \left[\left(1+\sqrt{I_{2} / I_{1}}\right) /\left(1-\sqrt{I_{2} / I_{1}}\right)\right]^{2}$, where $I_{1}$ and $I_{2}$ are the power distributed in the lower-order and the higher-order interference modes, respectively, with $I_{2}<I_{1}$. The extinction ratio is related to the power ratio $I_{2} / I_{1}$ of the two modes and decreases with the reduction of the power ratio [5]. Note that rotation or twist of the DCF will lead to the variation of the interference spectrum, which is mainly because part of the power in the dominant interference modes is coupled into other weak modes. In order to avoid the influence of rotation or twist of the DCF on the measurement result, the DCF is supported on an optical table by a fiber holder and a translation stage, which provides the longitudinal strain to the sensor head; moreover, the sensing DCF and a supporting DCF (identical to the sensing DCF) are fixed between two aluminum flakes with smooth surfaces, which provide the transverse strain to the sensor head, as shown in Fig. 1 . The width of the aluminum flake is about $1 \mathrm{~cm}$.

\section{B. Sensing Principle}

When a transverse force applied to a short section of the DCF, the extinction ratio will decrease with the increase of the transverse force [5]. The transverse strain induced by the transverse force can be expressed as [8]

$$
\left\{\varepsilon_{x}, \varepsilon_{y}\right\}=\left\{\frac{\left(1+3 \gamma+2 \gamma^{2}\right)}{\pi E b l} F, \frac{\left(2 \gamma^{2}-\gamma-3\right)}{\pi E b l} F\right\},
$$

where $\varepsilon_{x}$ and $\varepsilon_{y}$ are the $x$ and $y$ components of the strain induced by the transverse force; $F$ is the force acting on the short section of DCF $l$; and $\gamma, b$, and $E$ are the Poisson's coefficient, outer radius, and Young's modulus of the fiber, respectively. For fused silica fiber, $E$ and $\gamma$ are about $6.5 \times 10^{-4} \mathrm{~N} / \mathrm{mm}^{2}$ and 0.17 , respectively. It can be seen that the transverse strain is proportional to the transverse force and can be indirectly applied by the transverse force.

When a longitudinal strain is applied to the DCF, the transmission spectrum of the DCF interferometer will shift, which is related to only the Poisson's ratio and effective strain-optic coefficient of the fiber [1-4]. The transverse force is applied to only a short section of DCF, which is much shorter than the whole length of the DCF. As the elongation of the DCF induced by the transverse force is far less than the short section, the resultant longitudinal strain in the short section is negligible; therefore, the interference spectrum produced by the whole DCF will not shift due to the applied transverse strain. Although the extinction ratio varies with the applied transverse force, it almost does not change with the variation of the longitudinal strain. This feature can be used to measure the transverse and longitudinal strain simultaneously.

The response of DCF interferometer to temperature is only related to the thermal expansion and thermo-optic coefficients of the fiber material [2-4]. The extinction ratio only varies with the applied 
lateral force, while it does not change with the temperature variation [5]. This feature can be applied to realize simultaneous measurement of transverse strain and temperature.

\section{Experimental Validation and Discussion}

\section{A. Response to Transverse and Longitudinal Strain}

Figure 2 shows the transmission power spectra of the sensor under different longitudinal strain at fixed transverse force of $0 \mathrm{~N}$ and $2.94 \mathrm{~N}$. When the longitudinal strain increases, the transmission spectrum of the sensor has a blueshift. It can be seen that the longitudinal strain only leads to the wavelength shift of the transmission spectra of the sensor and almost does not affect the extinction ratio. Figure 3 shows the relationship between the measured wavelength (marked with an arrow in Fig. 2) and the longitudinal strain under fixed transverse force of $0 \mathrm{~N}$ and $2.94 \mathrm{~N}$. It can be seen that there is a good linear relationship between the wavelength and the longitudinal strain under $0 \mathrm{~N}$ transverse force, and the correlation coefficient square and the longitudinal strain sensitivity are 0.9987 and $0.82 \times 10^{-3} \mathrm{~nm} / \mu \varepsilon$, respectively. The longitudinal strain resolution is about $12 \mu \varepsilon$ for the OSA with a $10 \mathrm{pm}$ resolution. The response for nonzero transverse force also has a good linear relationship. For the same longitudinal strain, the measured wavelengths at different fixed transverse forces are slightly different; the maximum wavelength deviation is less than $0.02 \mathrm{~nm}$ at zero longitudinal strain. Experimental results show that when the longitudinal strain is larger than $108 \mu \varepsilon$, the maximum slope difference under different transverse forces is less than $1 \times 10^{-5} \mathrm{~nm} / \mu \varepsilon$, and the wavelength deviation induced by the transverse force becomes negligible, as shown in Fig. 3. Such a small crosstalk can be relieved by using a preapplied longitudinal strain.

Figure 4 shows the transmission spectral response to different transverse forces at a fixed longitudinal strain of $0 \mu \varepsilon$ and $432 \mu \varepsilon$. It can be seen that when the transverse force increases, the extinction ratio de-

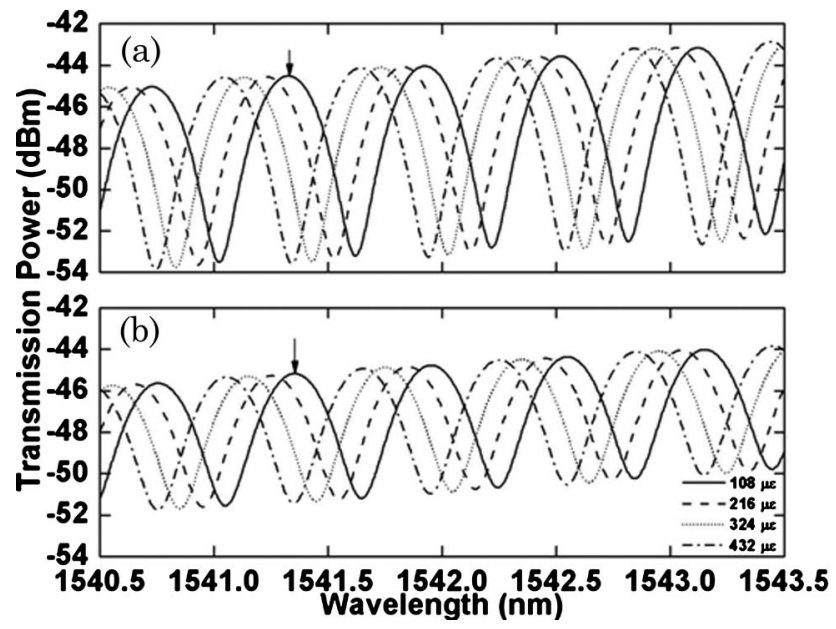

Fig. 2. Transmission spectral response to different longitudinal strains at a fixed transverse force of (a) $0 \mathrm{~N}$ and (b) $2.94 \mathrm{~N}$.

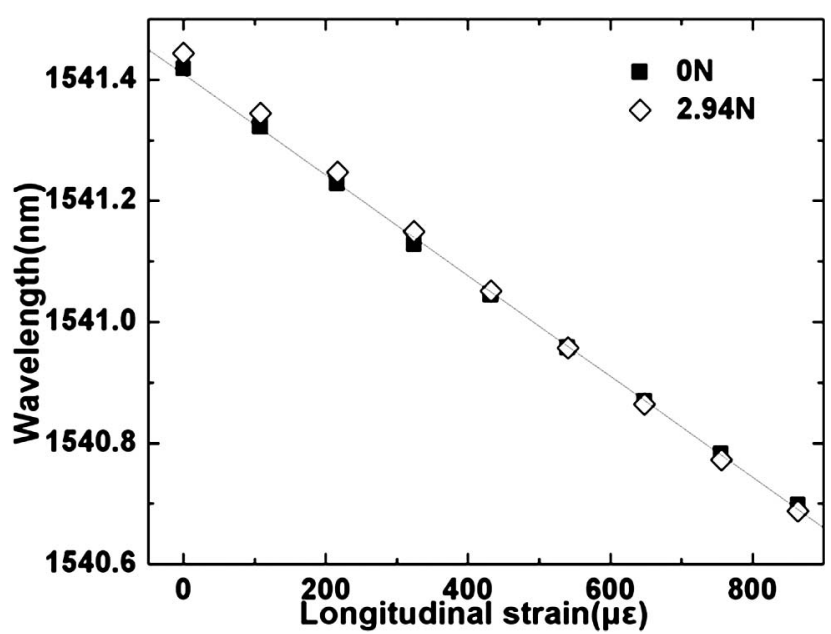

Fig. 3. Relationship between the wavelength and the longitudinal strain under fixed transverse force of (a) $0 \mathrm{~N}$ and (b) $2.94 \mathrm{~N}$.

creases accordingly, and the interference phase of the DCF interferometer is almost unaffected. Figure 5 shows the relationships between the measured extinction ratio (between the two marked arrows shown in Fig. 4) and the transverse strain $\varepsilon_{x}$ and $\varepsilon_{y}$ induced by the transverse force at a fixed longitudinal strain of $0 \mu \varepsilon$ and $432 \mu \varepsilon$, respectively. There is a good quadratic relationship between the extinction ratio and the transverse strain under zero longitudinal strain. The response for nonzero longitudinal strain also has a good quadratic relationship. For the same transverse strain, the measured extinction ratios at different fixed longitudinal strains are slightly different; the maximum extinction ratio deviation is less than $0.1 \mathrm{~dB}$, which has negligible influence on the measured quadratic relationship shown in Fig. 5. Note that the transverse strain resolution is related to amplitude noise, which might arise from the fluctuation of the BBS or the accuracy of the power measured by the OSA. In addition, the transverse strain sensitivity increases with an increase of the transverse strain; thus, the resolution is different at

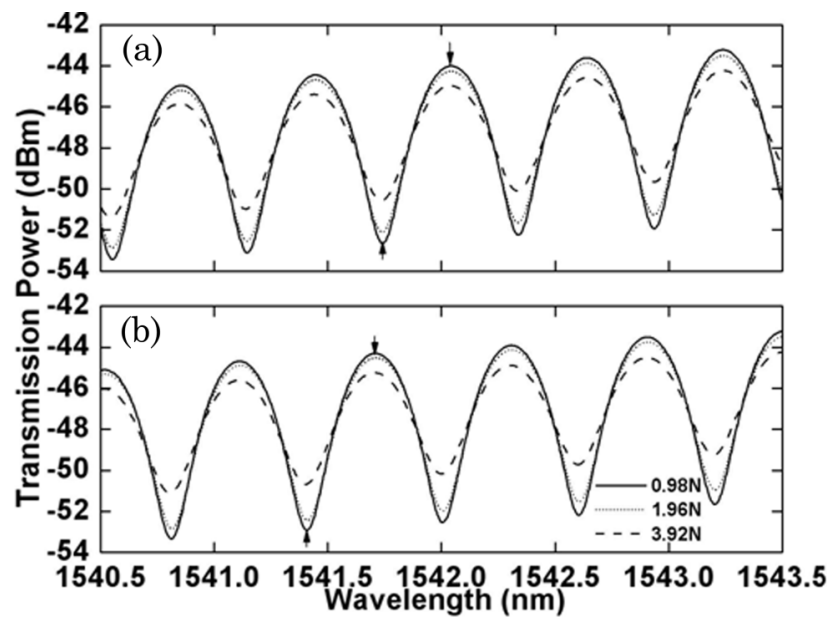

Fig. 4. Transmission spectral response to different transverse forces at a fixed longitudinal strain of (a) $0 \mu \varepsilon$ and (b) $432 \mu \varepsilon$. 


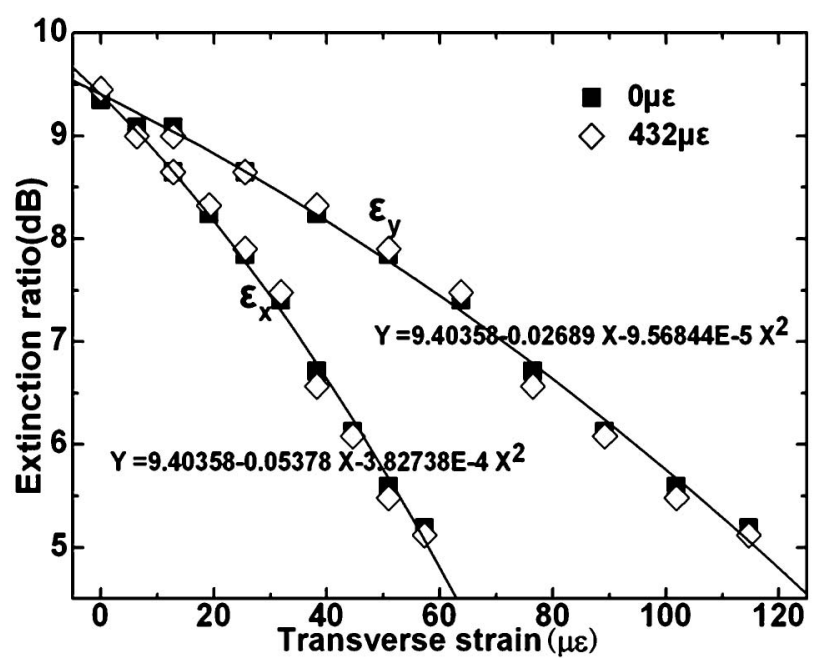

Fig. 5. Relationship between the extinction ratio and the transverse strain at a fixed longitudinal strain of (a) $0 \mu \varepsilon$ and (b) $432 \mu \varepsilon$.

different transverse strain. Experimental results show that an extinction ratio response can be observed by applying a minimum transverse strain of $3.18 \mu \varepsilon$. In order to avoid breaking the DCF, the applied transverse force is controlled in the range of $0-4.41 \mathrm{~N}$, resulting in transverse strain $\varepsilon_{x}$ and $\varepsilon_{y}$ in the range of $0-57.3 \mu \varepsilon$ and $0-114.66 \mu \varepsilon$, respectively. The applied longitudinal strain is controlled in the range of $0-864 \mu \varepsilon$. The measurement ranges of $\varepsilon_{x}, \varepsilon_{y}$, and $\varepsilon_{z}$ can be increased by encapsulating the DCF sensing head in materials with a high Young's modulus for practical applications.

\section{B. Response to the Temperature and Transverse Force}

If only the transverse force is applied to the short section of the DCF, the spectrum of the interferometer will have a redshift with an increase of the ambient temperature, as shown in Fig. $\underline{6}$. There is a good linear relationship between the wavelength and temperature, as shown in Fig. 7. The sensitivity reaches $0.058 \mathrm{~nm} /{ }^{\circ} \mathrm{C}$. However, the measured extinction ratio (between the two arrow marks in Fig. $\underline{6}$ ) is

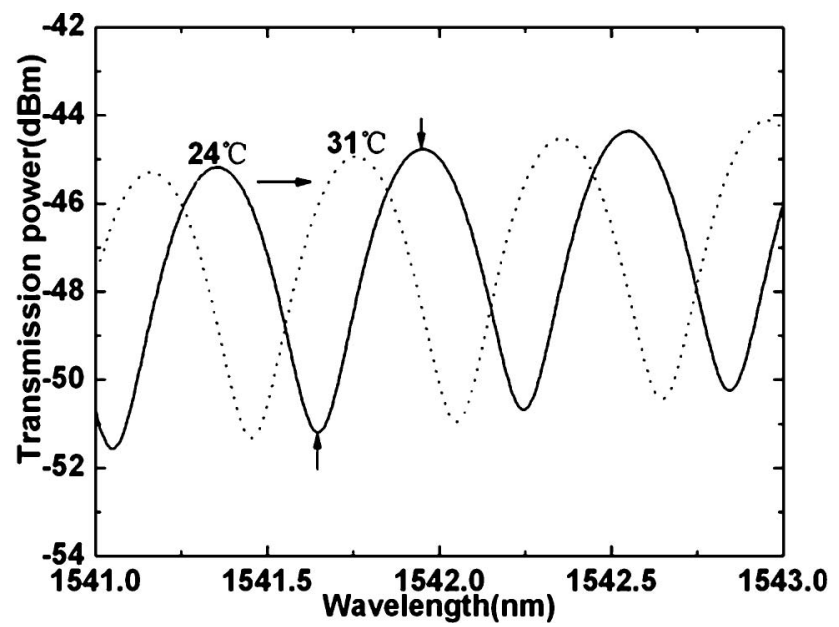

Fig. 6. Transmission spectral response to temperature under transverse force of $2.94 \mathrm{~N}$.

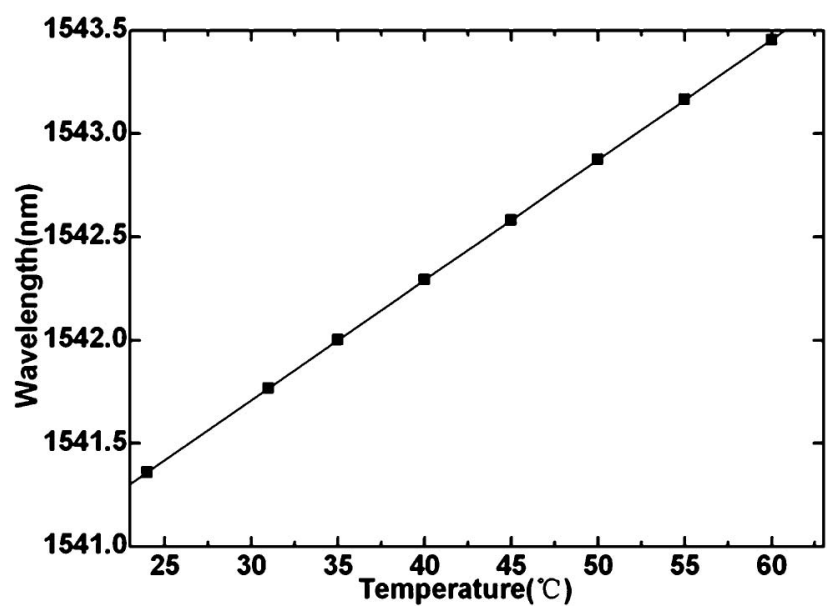

Fig. 7. Relationship between the wavelength and temperature under transverse force of $2.94 \mathrm{~N}$.

almost unaffected, and the extinction ratio fluctuation is less than $0.05 \mathrm{~dB}$ for a given transverse force, as shown in Fig. 8. The above phenomenon can be used to realize simultaneous measurement of transverse strain and temperature. Additional experiment results show that the extinction ratio response to temperature has variations without applying transverse strain when the temperature is over $60^{\circ} \mathrm{C}$. This is because the core diameter and the length of the DCF will have a relatively large variation under higher temperature, which will have corresponding influences on the coupling of the interference modes.

The above experimental results just agree with our theoretical predictions. The DCF interferometer can be used to measure both transverse and longitudinal strains, or both transverse strain and temperature, simultaneously with low crosstalk. In addition, if it is used as a two-directional (transverse and longitudinal) strains sensor, the sensing head can be encapsulated in temperature compensation materials with negative temperature coefficient in order to reduce the temperature-strain cross effect.

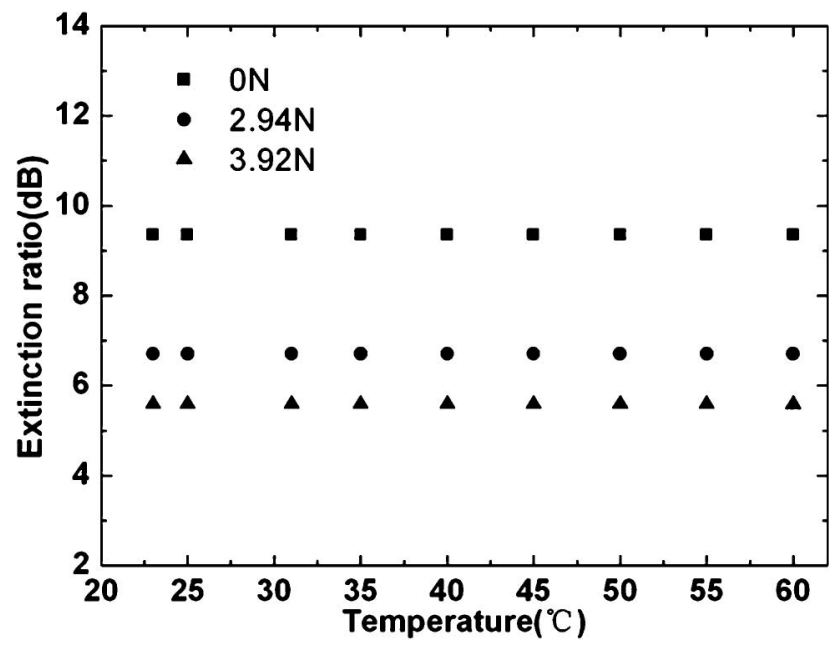

Fig. 8. Measured extinction ratio response to temperature. 


\section{Conclusions}

We propose a novel core-offset DCF interferometer and investigate its application in fiber sensors. Due to the little crosstalk between the extinction ratio change induced by the transverse force and the wavelength shift induced by the longitudinal strain variations or temperature variations, which can be used to realize simultaneous measurement of transverse and longitudinal strain or simultaneous measurement of transverse strain and temperature. The proposed sensors have the advantages of low cost, simple and compact structure, and good reproducibility.

This work is supported by the Natural Science and Engineering Research Council of Canada (NSERC). The authors thank Sumitomo Electric Industries, Ltd., for providing the $1.9 \mu \mathrm{m} / 115.7 \mu \mathrm{m}$ DCF.

\section{References}

1. C. Belleville and G. Duplain, "White-light interferometric multimode fiber-optic strain sensor," Opt. Lett. 18, 78-80 (1993).
2. Y. Liu and L. Wei, "Low-cost high-sensitivity strain and temperature sensing using graded-index multimode fibers," Appl. Opt. 46, 2516-2519 (2007).

3. E. Li, "Sensitivity-enhanced fiber-optic strain sensor based on interference of higher order modes in circular fibers," IEEE Photon. Tech. Lett. 19, 1266-1268 (2007).

4. D.-P. Zhou, L. Wei, W. K. Liu, Y. Liu, and J. W. Y. Lit, "Simultaneous measurement for strain and temperature using fiber Bragg gratings and multimode fibers," Appl. Opt. 47, 1668-1672 (2008).

5. B. Dong, D.-P. Zhou, L. Wei, W.-K. Liu, and J. W. Y. Lit, "Temperature- and phase-independent lateral force sensor based on a core-offset multi-mode fiber interferometer," Opt. Express 16, 19291-19296 (2008).

6. B. G. Grossmann and L.-T. Huang, "Fiber optic sensor array for multi-dimensional strain measurement," Smart Mater. Struct. 7, 159-165 (1998).

7. L. Yuan, Q. Li, Y. Liang, J. Yang, and Z. Liu, "Fiber optic 2-D sensor for measuring the strain inside the concrete specimen," Sens. Actuators A, Phys. 94, 25-31 (2001).

8. R. B. Wagreich, W. A. Atia, H. Singh, and J. S. Sirkis, "Effects of diametric load on fibre Bragg gratings fabricated in low birefringent fibre," Electron. Lett. 32, 1223-1224 (1996). 